\title{
Effectiveness and safety of exenatide in Korean patients with type 2 diabetes inadequately controlled with oral hypoglycemic agents: an observational study in a real clinical practice
}

\author{
You-Cheol Hwang ${ }^{1}$, Ari Kim², Euna Jo ${ }^{2}$, Yeoree Yang ${ }^{3}$, Jae-Hyoung Cho ${ }^{3^{*+}}$ and Byung-Wan Lee ${ }^{4^{*+}+}$
}

\begin{abstract}
Background: Randomized clinical trials have shown the efficacy and safety of short-acting exenatide in patients with type 2 diabetes mellitus (T2DM). The aim of this observational study was to investigate the effectiveness and safety of exenatide twice a day in Korean patients with T2DM who are suboptimally controlled with oral hypoglycemic agents.

Methods: This study was a post hoc analysis of multi-center (71 centers), prospective, observational, single-arm, postmarketing study of short-acting exenatide 5 to $10 \mu$ g twice a day from March 2008 to March 2014 and analyzed those who finished the follow-up over 20 weeks of medication. Changes of hemoglobin A1c ( $\mathrm{HbA1c}$ ), fasting plasma glucose $(F P G)$, and body weight values before and after exenatide treatment were analyzed. Adverse events and adverse drug reactions were estimated in patients who were treated with exenatide at least once and for whom follow-up for safety has been completed.
\end{abstract}

Results: After 20 weeks treatment with exenatide, mean $\mathrm{HbA1c}$ and body weight were significantly reduced from 8.4\% to $7.7 \%$ and from $83.4 \mathrm{~kg}$ to $80.2 \mathrm{~kg}$, respectively (both $p<0.001$ ). Subjects with higher baseline glucose and HbA1c levels showed an independent association with a greater reduction in glucose level. In addition, short duration of diabetes less than 5 years was an independent predictor for the improvement in glucose level. The majority of study subjects showed a reduction in both body weight and glucose level (63.3\%) after exenatide treatment. In terms of safety profile, exenatide treatment was generally well-tolerated and the incidence of severe adverse event was rare $(0.8 \%)$. The gastrointestinal side effects were most common and hypoglycemia was reported in $1.7 \%$ of subjects.

Conclusion: In real clinical practice, 20 weeks treatment with short-acting exenatide was well tolerated and showed a significant body weight and glucose reduction in Korean patients with T2D who are suboptimally controlled with oral hypoglycemic agents.

Trial registration: ClinicalTirals.gov, number NCT02090673, registered 14 February 2008.

Keywords: Exenatide, Type 2 diabetes mellitus, Glucose, Adverse event, GLP-1 analogue

\footnotetext{
* Correspondence: drhopper@ikoob.com; bwanlee@yuhs.ac

${ }^{\dagger}$ Equal contributors

${ }^{3}$ Division of Endocrinology and Metabolism, Department of Internal Medicine, Seoul St. Mary's Hospital, College of Medicine, The Catholic University of Korea, 222 Banpo-daero, Seoul, Seocho-gu 06591, South Korea

${ }^{4}$ Division of Endocrinology and Metabolism, Department of Internal

Medicine, Severance Hospital, Yonsei University College of Medicine, 50-1

Yonsei-Ro, Seoul, Seodaemun-Gu 03722, South Korea

Full list of author information is available at the end of the article
}

\section{$\int$ Biomed Central}

(c) The Author(s). 2017 Open Access This article is distributed under the terms of the Creative Commons Attribution 4.0 International License (http://creativecommons.org/licenses/by/4.0/), which permits unrestricted use, distribution, and reproduction in any medium, provided you give appropriate credit to the original author(s) and the source, provide a link to the Creative Commons license, and indicate if changes were made. The Creative Commons Public Domain Dedication waiver (http://creativecommons.org/publicdomain/zero/1.0/) applies to the data made available in this article, unless otherwise stated. 


\section{Background}

The prevalence of obesity and type 2 diabetes mellitus (T2DM) is now increasing rapidly in Korea and Asian countries [1-3]. However, glycemic control is not satisfactory and the proportions of patients with reaching target hemoglobin A1c (HbA1c) of $<6.5 \%$ and $<7.0 \%$ were $27 \%$ and $45.6 \%$, respectively in Korea [4]. In addition, different side effects related to oral hypoglycemic agents including weight gain and hypoglycemia [5] can cause a nonadherence to medications and poor glycemic control [6]. Therefore, there is still unmet need for another class of hypoglycemic agent has both good efficacy and safety.

Exenatide is an incretin mimetic that activates glucagon-like-peptide-1 (GLP-1) receptors. It mainly decreases postprandial plasma glucose level by stimulating insulin secretion, suppressing glucagon secretion and delaying gastric emptying in a glucose-dependent manner $[7,8]$. Numerous clinical studies in Western countries have shown the glucose-lowering efficacy of exenatide monotherapy [9] and in combination with other oral hypoglycemic agents including sulfonylurea, metformin, and thiazolidinediones [10-13].

However, the pathophysiology of T2DM could be different according to ethnicity and it was reported that $\beta$-cell dysfunction is a main pathogenic mechanism for the development of T2DM in Asian population but, a lesser degree of insulin resistance compared with Caucasian [1, 14]. In addition, glucose-lowering effect of GLP-1 analogues was reported to be greater in Asian-dominant studies than in non-Asian-dominant studies [15]. Furthermore, it has been well known that results from real clinical setting are different from those from randomized clinical trial (RCT) and thus, observational studies are also necessary to evaluate the effectiveness of health care [16].

Therefore, the aim of this study was to investigate the effects of exenatide twice a day injection in a real clinical practice on blood glucose level, body weight, and safety profiles in obese Korean patients with T2DM who are suboptimally controlled with oral hypoglycemic agents.

\section{Methods}

\section{Study design}

This is a post hoc analysis of multi-center (71 centers), prospective, observational, single-arm, post-marketing surveillance (PMS) study to evaluate the safety and effectiveness of short-acting exenatide (Byetta ${ }^{\circ}$ ), conducted from March 2008 to March 2014, in patients with T2DM (ClinicalTrials.gov, number NCT02090673). The investigators' decisions regarding the proper treatment and care of the patient were made in the course of the normal clinical practice; without blinding or randomization to particular comparator arms or therapies. In this study, patients were enrolled for the collection of their data on observations made during normal clinical practice such as serum HbA1c, fasting plasma glucose (FPG), body weight, medical history, and adverse events.

\section{Study populations and treatments}

The patient population for this study consisted of Korean patients who were at least 18 years old, diagnosed with T2DM, and were treated with short-acting exenatide in an ambulatory care setting according to the approved label. Patients were excluded from the study for any of the following reasons; simultaneously participating in a different study that includes a treatment intervention and/or an investigational drug, being pregnant or having intentions to be pregnant within the duration of the study, and being contraindicated for short-acting exenatide. Investigators have ensured that patients, or, in those situations where consent could not be given by patients, their legally acceptable representatives, are clearly and fully informed about the purpose of the study, potential risks, the patient's rights and responsibilities when participating in this study.

All patients were initially injected using $5 \mu \mathrm{g}$ of shortacting exenatide twice daily. After 1 month of injection $5 \mu \mathrm{g}$, the exenatide dosage was intended to increase to $10 \mu \mathrm{g}$ twice a day under the decision of the physician. The previous antidiabetic medications were maintained during the periods of exenatide treatment. Life style modification therapy and education for not only diet but also exercise were combined in parallel with medications.

\section{Effectiveness assessment}

Based on electronic database of the study with high confidentiality, the patients were rearranged for the purpose of reanalysis settings (Fig. 1). The variables for the effectiveness of short-acting exenatide after 20 weeks of treatment were changes in HbA1c, FPG, body mass index (BMI), and body weight. Total 1269 patients who received more than one-time injection of exenatide were reviewed. Of these, 181 and 334 patients dropped out within 8 and 20 weeks, respectively. Thus, 754 patients continued short-acting exenatide over 20 weeks and were reviewed for safety analysis. With additional exclusion of 32 patients due to lack of clinical data, a final number of 722 patients were reviewed for effectiveness analysis. Among them, 619 patients who measured HbA1c level both at baseline and 20-week endpoint were analyzed for HbA1c. In the same way, 540, 655, and 674 patients were analyzed for FPG, BMI, and body weight, respectively. Patients were also defined by 2dimensional quadrant allocation according to the absolute change in HbA1c and body weight in the same picture.

Reanalysis for the effectiveness was conducted using stratified categorization with the responsiveness for the short-acting exenatide. In the first stratum by median 


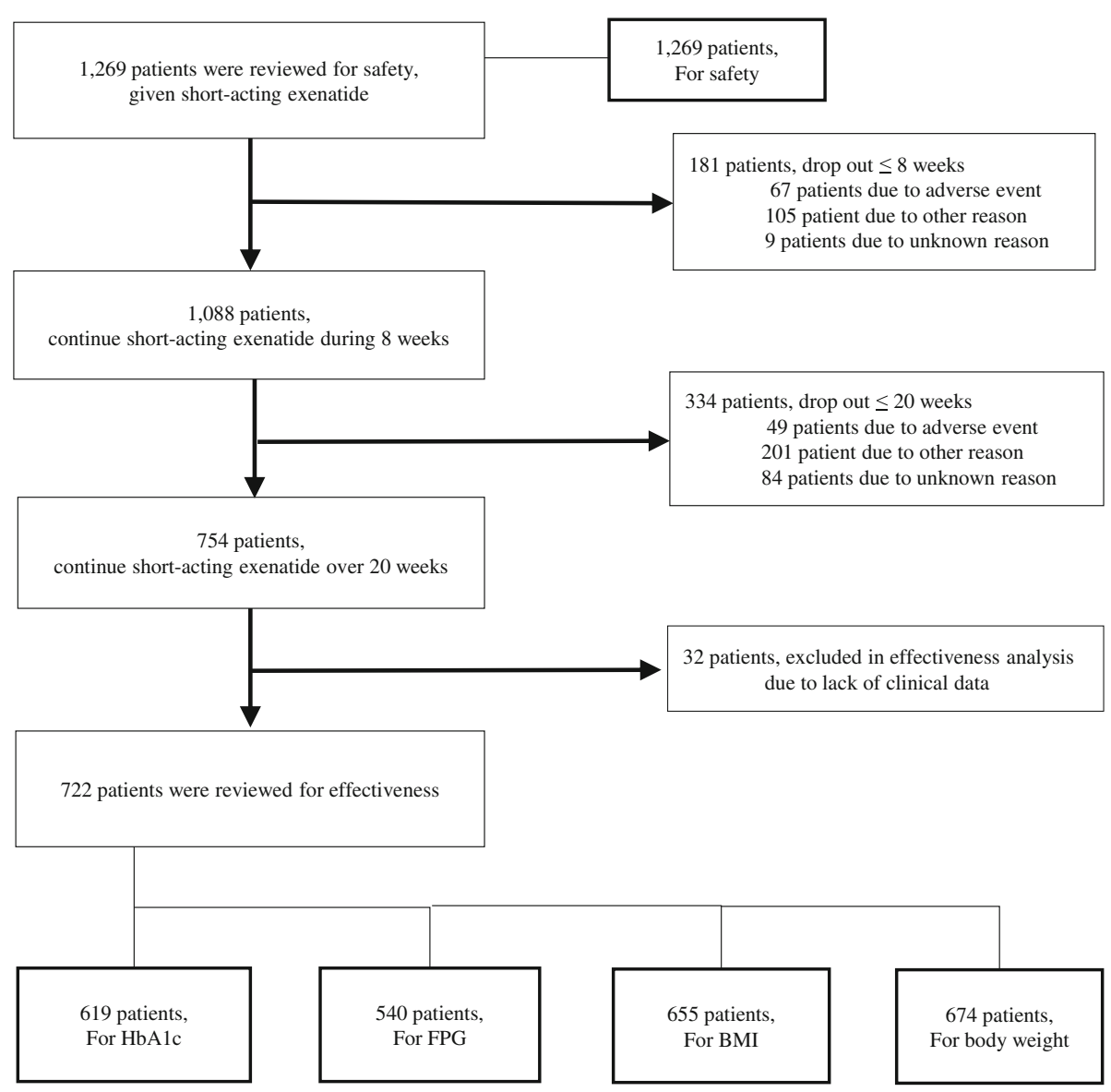

Fig. 1 Study flow diagram demonstrating analysis population

value of $\mathrm{HbA1c}$ reduction, patients were divided into two groups with $0.6 \% \mathrm{HbA1c}$ reduction. The second stratum used the absolute value as target $\mathrm{HbAlc}$ as $7.5 \%$. As known in various randomized controlled trials, mean $\mathrm{HbA} 1 \mathrm{c}$ at the end of treatment, the primary endpoint in the majority of studies, was decreased by 0.79 to $1.75 \%$ (the median value across these large studies was $1.11 \%$ ) [17]. The baseline HbA1c of our study was $8.4 \%$ and we assumed responder as patients who would reach $\mathrm{HbA1c}$ level at $7.5 \%$ after 20 weeks of exenatide treatment. On the other hands, the third stratum was applied by the mean change percentage of HbA1c from baseline which was calculated as $9.8 \%$ as percentage. The mean $\mathrm{HbA1c}$ level was $8.40 \%$ before treatment and $7.56 \%$ after exenatide over 20 weeks. The absolute difference was $0.84 \%$, which was re-calculated as $9.8 \%$ as percentage compared to basal mean HbA1c level.

\section{Safety assessment}

Patients who were participating the study could reject or withdraw the continuation of treatment or the study with the reason of follow-up lost, adverse events, patient's decision, or physician's decision. If a patient discontinued the treatment during the study, the patients were contacted to collect all adverse events information and reasons why they want to stop the treatment. All relevant information was captured by data form. Patients who were discontinued from study were included to safety population if they had taken at least one dose of exenatide. We calculated the discontinuation rate due to any adverse events. Adverse events and serious adverse events which were recorded in electronic case report form were recoded and reanalyzed according to the current routine classification. For the serious adverse events, we reviewed the patient record and revealed the diagnosis of them.

\section{Statistical analysis}

All statistical analysis was performed with SAS (version 9.4, SAS Institute, Cary, NC, USA) for the numerics, and NCSS (version 11, NCSS company, Kaysville, UT, USA) for the graphics. For effectiveness analysis, missing values was imputed using the last observation carried forward methods. Baseline characteristics were shown in descriptive statistics such as mean and standard deviation for continuous data, or frequency and percentage 
for categorical data. Safety analysis was also summarized descriptively.

Clinical factors which could affect the HbA1c reduction between responder and non-responder groups were analyzed using chi-square test in 3 different strata. We performed multivariate logistic regression test to adjust the potential variables for the HbA1c reduction in each stratum. To confirm the correlation between changes of HbA1c and body weight, scattered graph was rendered and correlation coefficient was calculated. If $p$-value is less than 0.05 , it was considered statistically significant.

\section{Results}

\section{Baseline clinical and laboratory characteristics of participants}

Of the total 1269 patients who received more than onetime injection of exenatide twice a day, data retrieved from 754 patients who completed 20 weeks treatment with exenatide were analyzed for the safety profiles (safety analysis set). Among the 754 participants, 619 patients who measured HbA1c level both at baseline and 20-week endpoint were analyzed for the effectiveness (effectiveness analysis set).

Based on the safety analysis set $(n=754)$, the mean age and BMI of study subjects were 48.8 years and $31.2 \mathrm{~kg} / \mathrm{m}^{2}$, respectively. $34.0 \%$ of the subjects were men. Approximately one third of the study subjects had a family history of diabetes and duration of diabetes was 7.7 years. At baseline, mean HbA1c and FPG level were $8.4 \%$ and $164.3 \mathrm{mg} / \mathrm{dL}$, respectively (Table 1 ).

\section{Clinical and laboratory variables affecting glucose lowering effectiveness}

In the current study, mean dosage of exenatide was $16.0 \mu \mathrm{g} /$ day. A significant reduction in FPG $(15.12 \mathrm{mg} / \mathrm{dL})$ and $\mathrm{HbAlc}(-0.75 \%)$ levels from baseline were observed over 20-week of exenatide treatment. To determine glucose-lowering effect of exenatide, we used three different criteria to define responder to exenatide treatment. The characteristics of responders by three definitions as stratum are shown in Table 2. Using a responsiveness based on absolute value of $\mathrm{HbA} 1 \mathrm{c}$ reduction $\geq 0.6 \%$, subjects with optimal response to exenatide had significantly higher baseline FPG and HbA1c levels than those with suboptimal response to exenatide treatment. In addition, men and subjects with short duration of diabetes less than 5 years were more likely to improve in glucose level with exenatide treatment with significance. However, age and BMI did not affect glucose-lowering effectiveness of exenatide treatment. Using a responsiveness based on achievement in HbA1c $\leq 7.5 \%$ at 20 -week endpoint, subjects who reached the target $\mathrm{HbA1c}$ had statistically lower baseline FPG and HbA1c levels. The subjects with short duration of diabetes showed significantly better glucose
Table 1 Baseline characteristics of the study subjects

\begin{tabular}{|c|c|}
\hline Baseline demographics & $\begin{array}{l}\text { Patients who completed at least } 20 \text { weeks } \\
\text { of short-acting exenatide }(n=754)\end{array}$ \\
\hline Age (years) & $48.76 \pm 11.44$ \\
\hline \multicolumn{2}{|l|}{ Sex } \\
\hline Male & $256(33.95)$ \\
\hline Female & $498(66.05)$ \\
\hline BMI $\left(\mathrm{kg} / \mathrm{m}^{2}\right)$ & $31.15 \pm 4.68$ \\
\hline Baseline HbA1c (\%) & $8.40 \pm 1.74$ \\
\hline Baseline FPG (mg/dL) & $164.26 \pm 62.10$ \\
\hline Daily dose $(\mu \mathrm{g})$ & $15.96 \pm 3.46$ \\
\hline \multicolumn{2}{|l|}{ Diabetes family history } \\
\hline No & $491(65.12)$ \\
\hline Yes & $263(34.88)$ \\
\hline $\begin{array}{l}\text { Duration of diabetes } \\
\text { (months) }\end{array}$ & $92.66 \pm 79.42$ \\
\hline \multicolumn{2}{|l|}{ Concurrent disease } \\
\hline No & $115(15.25)$ \\
\hline Yes & 639 (84.75) \\
\hline \multicolumn{2}{|l|}{ Antidiabetic medication } \\
\hline 0 or 1 medication & $260(34.48)$ \\
\hline 2 or more medications & $494(65.52)$ \\
\hline \multicolumn{2}{|c|}{ Kind of antidiabetic medications } \\
\hline Metformin & $672(89.12)$ \\
\hline Sulfonylureas & $494(65.52)$ \\
\hline Thiazolidinediones & $17(2.25)$ \\
\hline \multicolumn{2}{|c|}{ Other concomitant medication } \\
\hline No & $80(10.61)$ \\
\hline Yes & 674 (89.39) \\
\hline
\end{tabular}

Values are presented as mean \pm standard deviation of the mean or the number with percentage

Abbreviations: BMI Body Mass Index, HbA1c Hemoglobin A1c, Fasting plasma glucose

control. Using a responsiveness based on achievement in $\geq 9.8 \%$ of baseline HbA1c, subjects with optimal response to exenatide had significantly higher baseline FPG and HbA1c levels. Similar to analysis stratum 1, subjects with short duration of T2DM were more likely to improve in glucose level with exenatide treatment with significance. Before the initiation of exenatide, patients who had been taking 2 oral antidiabetics showed relatively higher proportion in responder in stratum classified by the changes from baseline. However, in the analysis of target HbA1c as $7.5 \%$, patients with 0 or 1 antidiabetics demonstrated better achievement proportion. To determine which clinical and laboratory variables were independently associated with glucose-lowering effectiveness of exenatide, multivariate logistic regression analysis was used. As a result, higher baseline FPG and HbA1c levels were independently associated with greater reduction in glucose level after 
Table 2 Clinical factors which may affect $\mathrm{HbA} 1 \mathrm{c}$ reduction after treatment with short-acting exenatide over 20 weeks

\begin{tabular}{|c|c|c|c|c|c|c|c|c|c|}
\hline & \multicolumn{3}{|c|}{$\mathrm{HbA} 1 \mathrm{c}$ reduction $\geq 0.6 \%$} & \multicolumn{3}{|l|}{$\mathrm{HbA} 1 \mathrm{c}<7.5 \%$} & \multicolumn{3}{|c|}{$\mathrm{HbA} 1 \mathrm{c}$ reduction $\geq 9.8 \%$ from baseline } \\
\hline & Yes $(n=316)$ & No $(n=303)$ & P & Yes $(n=346)$ & No $(n=273)$ & $P$ & Yes $(n=263)$ & No $(n=356)$ & $P$ \\
\hline \multicolumn{10}{|c|}{ Age (years) } \\
\hline$\geq 50$ & $24.4(151)$ & $24.9(154)$ & 0.45 & $27.1(168)$ & $22.1(137)$ & 0.69 & $20.0(124)$ & $29.2(181)$ & 0.36 \\
\hline$<50$ & $26.7(165)$ & $24.1(149)$ & & $28.8(178)$ & $22.0(136)$ & & $22.5(139)$ & $28.3(175)$ & \\
\hline \multicolumn{10}{|l|}{ Sex (\%) } \\
\hline Male & $19.9(123)$ & $13.6(84)$ & 0.003 & $19.7(122)$ & $13.7(85)$ & 0.28 & $17.3(107)$ & $16.2(100)$ & 0.001 \\
\hline Female & $31.2(193)$ & $35.4(219)$ & & $36.2(224)$ & $30.4(188)$ & & $25.2(156)$ & $41.4(256)$ & \\
\hline \multicolumn{10}{|c|}{ BMI $\left(\mathrm{kg} / \mathrm{m}^{2}\right)$} \\
\hline$\geq 30$ & $31.6(184)$ & $27.2(158)$ & 0.13 & $32.8(191)$ & $26.0(151)$ & 0.69 & $25.8(150)$ & $33.0(192)$ & 0.41 \\
\hline$<30$ & $19.6(114)$ & $21.7(126)$ & & $23.7(138)$ & $17.5(102)$ & & $16.7(97)$ & $24.6(143)$ & \\
\hline \multicolumn{10}{|c|}{$\mathrm{HbA1c}(\%)$} \\
\hline$\geq 8.5$ & 30.7 (190) & $13.6(84)$ & $<0.001$ & $12.1(75)$ & $32.2(199)$ & $<0.001$ & $25.5(158)$ & $18.7(116)$ & $<0.001$ \\
\hline$<8.5$ & $20.4(126)$ & 35.4 (219) & & $43.8(271)$ & $12.0(74)$ & & $17.0(105)$ & $38.8(240)$ & \\
\hline \multicolumn{10}{|c|}{ FPG (mg/dL) } \\
\hline$\geq 160$ & $27.9(137)$ & $15.0(74)$ & $<0.001$ & $17.1(84)$ & $25.8(127)$ & $<0.001$ & $23.0(113)$ & $19.9(98)$ & $<0.001$ \\
\hline$<160$ & $23.6(116)$ & $33.5(165)$ & & $41.1(202)$ & $16.1(79)$ & & $19.7(97)$ & $37.4(184)$ & \\
\hline \multicolumn{10}{|c|}{ Daily dose of exenatide ( $\mu \mathrm{g} /$ day) } \\
\hline$\geq 15$ & $36.5(226)$ & $33.9(210)$ & 0.55 & $39.6(245)$ & $13.3(82)$ & 0.82 & $31.3(194)$ & $39.1(242)$ & 0.12 \\
\hline$<15$ & $14.5(90)$ & $15.0(93)$ & & $16.3(101)$ & $30.9(191)$ & & $11.2(69)$ & $18.4(114)$ & \\
\hline \multicolumn{10}{|c|}{ Family history of diabetes (\%) } \\
\hline No & $28.4(135)$ & $24.2(115)$ & 0.96 & $29.1(138)$ & $23.6(112)$ & 0.86 & $23.6(112)$ & $29.1(138)$ & 0.68 \\
\hline Yes & $25.5(121)$ & $21.9(104)$ & & $26.5(126)$ & $20.8(99)$ & & $22.1(105)$ & $25.3(120)$ & \\
\hline \multicolumn{10}{|c|}{ Duration of diabetes (years) } \\
\hline$\geq 5$ & $29.4(158)$ & $32.0(172)$ & 0.040 & $26.3(141)$ & $35.2(189)$ & $<0.001$ & $23.8(128)$ & $37.6(202)$ & 0.023 \\
\hline$<5$ & $22.0(118)$ & $16.6(89)$ & & $26.8(144)$ & $11.7(63)$ & & $18.8(101)$ & $19.7(106)$ & \\
\hline \multicolumn{10}{|c|}{ Concurrent disease (\%) } \\
\hline No & $6.8(42)$ & $6.5(40)$ & 0.97 & $7.9(49)$ & $5.3(33)$ & 0.45 & $5.8(36)$ & $7.4(46)$ & 0.78 \\
\hline Yes & $44.3(274)$ & $42.5(263)$ & & $48.0(297)$ & $38.8(240)$ & & $36.7(227)$ & $50.1(310)$ & \\
\hline \multicolumn{10}{|c|}{ Number of previous antidiabetic medication (\%) } \\
\hline 0 or 1 & $15.5(96)$ & $19.1(118)$ & 0.025 & $24.2(150)$ & $10.3(64)$ & $<0.001$ & $12.8(79)$ & $21.8(135)$ & 0.042 \\
\hline 2 & $35.5(220)$ & $29.9(185)$ & & $31.7(196)$ & $33.8(209)$ & & $29.7(184)$ & $35.7(221)$ & \\
\hline \multicolumn{10}{|c|}{ Other concomitant medication (\%) } \\
\hline No & $3.7(23)$ & $5.2(32)$ & 0.15 & $5.3(33)$ & $3.6(22)$ & 0.52 & $3.4(21)$ & $5.5(34)$ & 0.50 \\
\hline Yes & $47.3(293)$ & $43.8(271)$ & & $50.6(313)$ & $40.6(251)$ & & $39.1(242)$ & $52.0(322)$ & \\
\hline
\end{tabular}

Abbreviations: BMI Body Mass Index, HbA1c Hemoglobin A1c , Fasting plasma glucose

Chi-square test

Data are expressed as percentage (number)

Body mass index, fasting plasma glucose, family history of diabetes, and duration of diabetes were determined in $582,492,475$, and 537 subjects, respectively

exenatide treatment. In contrast, subjects with longer duration of diabetes more than 5 years showed an inverse association with the improvement in glucose control (Table 3).

Efficacy of Exenatide for HbA1c level and weight changes We next determined the weight change with exenatide treatment. After 20 weeks treatment with exenatide twice a day, body weight was significantly reduced from $83.4 \mathrm{~kg}$ to $80.2 \mathrm{~kg}(p<0.001$, Fig. 2$)$. The majority of study participants showed a reduction in both body weight and glucose level after exenatide treatment (63.3\%) whereas, only $3.8 \%$ of subjects did not respond to exenatide treatment in both body weight and glucose aspects. Interestingly, $22.0 \%$ of subjects showed a weight reduction but, aggravation in glucose control and $11.0 \%$ 
Table 3 Adjusted potential variables for the prediction of the HbA1c responses according to the stratified classification

\begin{tabular}{|c|c|c|c|c|c|c|}
\hline & \multicolumn{2}{|c|}{$\mathrm{HbA} 1 \mathrm{c}$ reduction $\geq 0.6 \%$} & \multicolumn{2}{|l|}{$\mathrm{HbA} 1 \mathrm{c}<7.5 \%$} & \multicolumn{2}{|c|}{$\begin{array}{l}\mathrm{HbA} 1 \mathrm{c} \text { reduction } \geq 9.8 \% \text { from } \\
\text { baseline }\end{array}$} \\
\hline & OR $(95 \% \mathrm{Cl})$ & $P$ & OR $(95 \% \mathrm{Cl})$ & $P$ & OR $(95 \% \mathrm{Cl})$ & $P$ \\
\hline Male sex & $1.47(0.94-2.29)$ & 0.09 & Not determined & & $1.40(0.90-2.15)$ & 0.13 \\
\hline $\mathrm{HbA} 1 \mathrm{c} \geq 8.5 \%$ & $3.13(1.95-5.04)$ & $<0.001$ & $0.13(0.08-0.22)$ & $<0.001$ & $2.36(1.48-3.77)$ & $<0.001$ \\
\hline $\mathrm{FPG} \geq 160 \mathrm{mg} / \mathrm{dL}$ & $1.60(1.01-2.54)$ & 0.047 & $0.58(0.35-0.97)$ & 0.037 & $1.47(0.93-2.34)$ & 0.10 \\
\hline Duration of diabetes $\geq 5$ years & $0.997(0.995-1.000)$ & 0.053 & $0.994(0.991-0.997)$ & $<0.001$ & $0.997(0.994-1.000)$ & 0.043 \\
\hline Number of previous antidiabetic medication ( 2 vs. 0 or 1 ) & $1.07(0.67-1.71)$ & 0.79 & $0.91(0.52-1.58)$ & 0.74 & $1.28(0.80-2.05)$ & 0.31 \\
\hline
\end{tabular}

Abbreviations: OR odd ratio, HbA1c Hemoglobin A1c, FPG Fasting plasma glucose

Multivariate logistic regression test; References of dependent variables in each stratum, Group 2, Group B, and group II, respectively

of subjects showed an improvement in glucose control despite of weight gain (Fig. 3).

\section{Tolerability and adverse events of Exenatide}

Regarding the tolerability of exenatide, 181 and 334 subjects among total 1269 patients discontinued the injection of exenatide twice a day within 8-week and between 8 and 20 weeks of study period. Of 1269 patients, 116 patients stopped the injection of exenatide for adverse events. The final drop-out rate of short-acting exenatide just due to the adverse events was $9.14 \%$. Within 8 weeks of medication, discontinuation rate due to adverse events was 5.3\% (67 patients out of 1269 patients). Consecutively, the discontinuation rate due to adverse events between 8 and 20 weeks of medication showed 4.5\% (49 patients out of 1088 patients) (Fig. 1).

Based on the safety analysis set $(n=754)$ who completed 20 weeks treatment with exenatide, severe adverse event was very rare $(0.8 \%)$ but 173 cases $(22.9 \%)$ of adverse events were reported. The gastrointestinal side effects, mostly nausea, were the most common side effect and hypoglycemia was reported in $1.7 \%$ of subjects (Table 4). When it comes to serious adverse events, 6 cases were cholecystitis, hyperglycemia, rotator cuff syndrome, tendon disorder, diabetic skin ulcer, and cellulitis.

\section{Discussion}

To overcome many shortcomings of the classic antidiabetic drugs (ADDs) such as hypoglycemia and weight
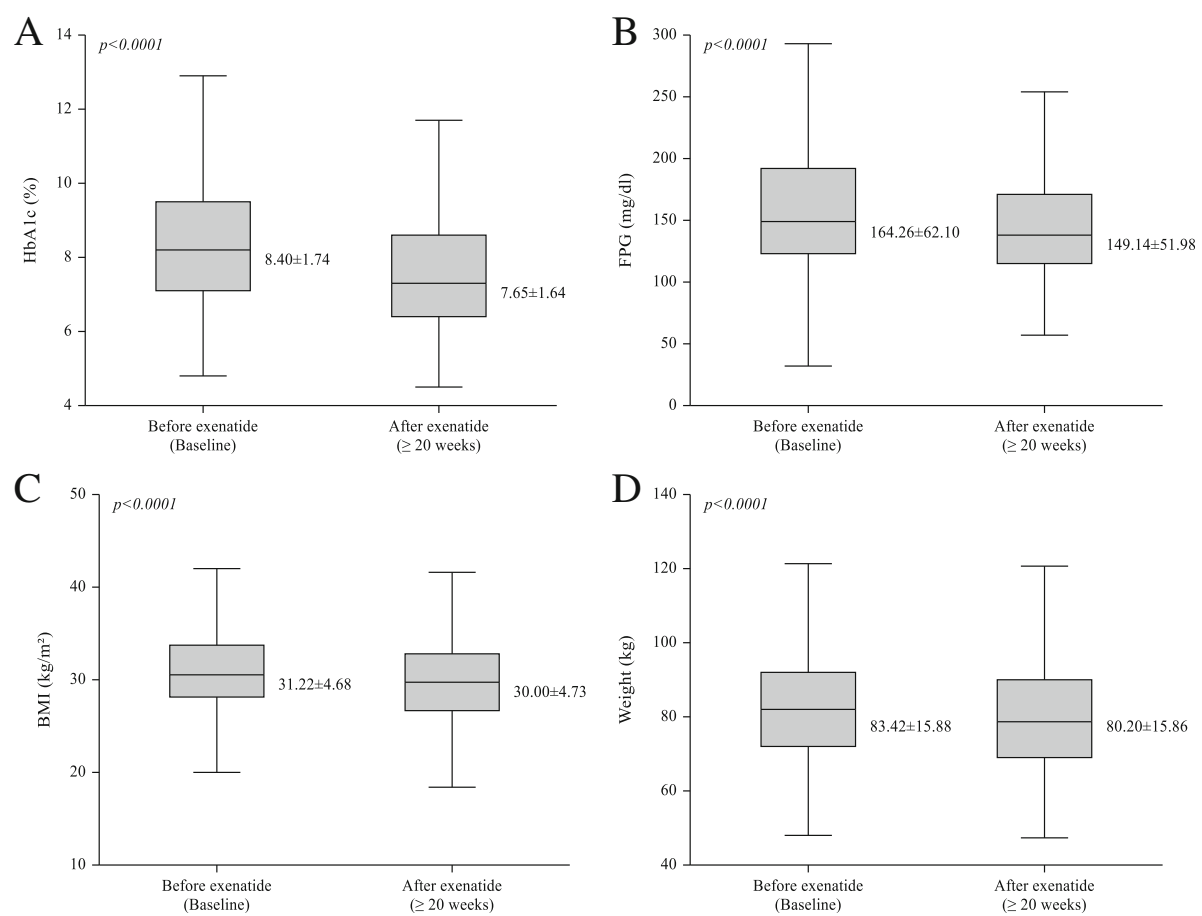

Fig. 2 Changes of clinical findings from before to after short-acting exenatide over 20 weeks. (A) Hemoglobin A1c (HbA1c) level ( $n=619)$, (B) Fasting plasma glucose (FPG) level $(n=54)$, (C) Body mass index (BMI) $(n=655)$, (D) Body weight $(n=674)$ 


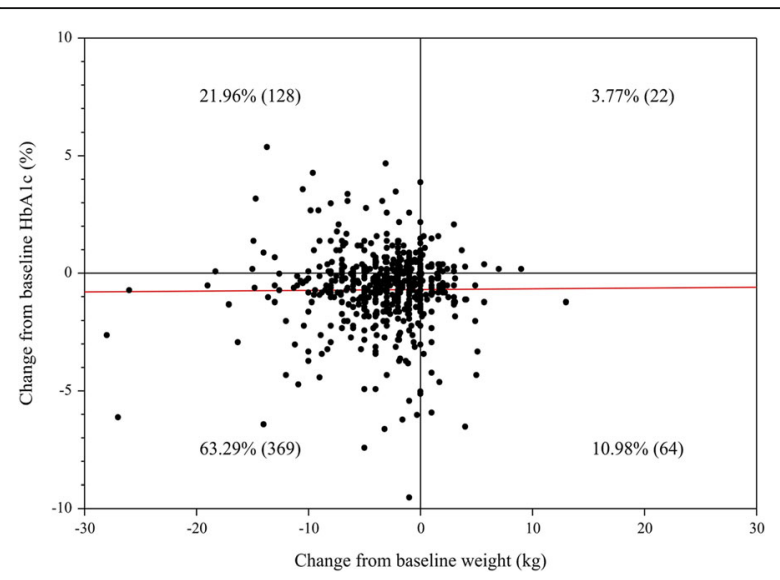

Fig. 3 Distribution of patients for the relation between Hemoglobin $\mathrm{A} 1 \mathrm{c}(\mathrm{HbA} 1 \mathrm{C})$ and body weight before and after treatment with short-acting exenatide over 20 weeks. Red line represents regression for $\mathrm{HbA} 1 \mathrm{c}$ according to body weight change, which shows the correlation coefficient as 0.00357 closed to 0 . It means that there is rare correlation between changes $\mathrm{HbA} 1 \mathrm{c}$ and body weight

Table 4 Summary of adverse events for the treatment with short-acting exenatide

\begin{tabular}{|c|c|c|}
\hline Adverse events & $\begin{array}{l}\text { Number of patients } \\
(\mathrm{n}=754)\end{array}$ & $\begin{array}{l}\text { Number of } \\
\text { events }\end{array}$ \\
\hline Any adverse events & $173(22.94 \%)$ & 246 \\
\hline Serious adverse events & $6(0.80 \%)$ & 6 \\
\hline Gastro-intestinal adverse events & $116(15.38 \%)$ & 154 \\
\hline Nausea & $93(12.33)$ & 100 \\
\hline Vomiting & $19(2.52 \%)$ & 19 \\
\hline Dyspepsia & $8(1.06 \%)$ & 8 \\
\hline Constipation & $7(0.93 \%)$ & 7 \\
\hline Diarrhoea & $5(0.66 \%)$ & 5 \\
\hline Others & $13(1.72 \%)$ & 15 \\
\hline $\begin{array}{l}\text { Metabolic and endocrine adverse } \\
\text { events }\end{array}$ & $22(2.92 \%)$ & 23 \\
\hline Hypoglycaemia & $13(1.72 \%)$ & 14 \\
\hline Others & $9(1.19 \%)$ & 9 \\
\hline Neuropsychologic adverse events & $25(3.32 \%)$ & 29 \\
\hline Dizziness & $7(0.93 \%)$ & 7 \\
\hline Headache & $6(0.80 \%)$ & 6 \\
\hline Anorexia & $5(0.66 \%)$ & 5 \\
\hline Others & $9(1.19 \%)$ & 11 \\
\hline Cardiovascular adverse events & $5(0.66 \%)$ & 5 \\
\hline Respiratory system adverse events & $3(0.40 \%)$ & 3 \\
\hline Urinary system adverse events & $1(0.13 \%)$ & 1 \\
\hline $\begin{array}{l}\text { Musculo-skeletal or generalized } \\
\text { adverse events }\end{array}$ & $16(1.21 \%)$ & 18 \\
\hline Others & $11(1.46 \%)$ & 13 \\
\hline
\end{tabular}

Data are expressed as percentage (number) gain etc., incretin-based drugs including GLP-1 analogues and dipeptidyl peptidase-4 (DPP4) inhibitors have been progressively developed and been regarded as a recommended drug for glycemic control in patients with T2DM [8]. Furthermore, by overcoming drug delivery system, long-acting GLP-1 s are now gaining popularity as innovative drug in managing the subjects with T2DM. Because of plentiful accumulated reports on exenatide, the first-inclass drug of GLP-1 analogue, the debate on clinical relevance of exenatide might be little. However, the clinical relevance on effectiveness and safety are reported mainly under the designed trials with the statistical expectations. We hypothesized that reluctance might remain to generalize the results of RCTs retrieved from a specific segment of the population to broader population or real practice $[16,18]$, and that it might be more important to both patients and physicians in managing the diabetes after identifying the results of RCTs with those of observation studies (OSs) retrieved from routine care. Based on these hypotheses, we aimed this study for investigating clinical effectiveness and safety of exenatide twice a day in real-life setting.

From this prospective, 20-week observational, phase 4 PMS study with short-acting exenatide twice a day in Korean patients with T2DM, we demonstrated 3 main findings. First, exenatide twice a day significantly induced HbA1c and weight reduction in real clinical practice. Second, higher baseline glucose level and short duration of diabetes were independent predictor for good glycemic control after exenatide treatment. Third, adverse events of exenatide in real-life setting are similar to those of RCTs [19].

With respect to study design, this analysis retrieved from the prospective, observational, PMS study. RCTs and OSs have both benefits and drawbacks reciprocally, which should be interpreted with balanced stance. Briefly, the inclusion and exclusion criteria of RCTs tend to be defined narrowly to conduct either the regulatory requirements in drug trials or the specific aims targeting selected population. The characteristics of patients who participate in the clinical trials are to be more concerned on their health status and care by health providers, and compliant with the drugs. In these regards, they might provide limited usefulness on the effectiveness and safety of a drug to generalize to the real world practice. In contrast, the design of PMS OSs in everyday practice setting is able to cover a broader population without stringent inclusion/exclusion criteria, and characteristics of patients. Furthermore, it also provides valuable information on the physician's preference and ideas [20]. These unmet need of RCTs but complemented by OSs as part of routine care might be important to both patients and health providers for treatment decisions.

With respect to the glycemic effectiveness of exenatide twice a day in real-life setting, exenatide improved 
glycemic control through up-regulating $\beta$-cells and down-regulating $\alpha$-cells residing in pancreatic islets. In recent meta-analysis, short-acting exenatide twice a day was effective in reducing HbA1c $(-0.61 \%$ for $5 \mu \mathrm{g}$, and - $0.83 \%$ for $10 \mu \mathrm{g}$ ) compared with placebo in patients with T2DM [21]. Furthermore, Kim et al. reported that GLP-1 analogues including exenatide, and liraglutide lowered HbA1c more in Asian-dominant studies than in non-Asian-dominant studies [15]. In this OS, there was significant reduction in both FPG $(-15.12 \mathrm{mg} / \mathrm{dL})$, and HbA1c $(-0.75 \%)$ from baseline. Regarding the responders to ADDs, the conventional definitions of responders might be one of the two analyses, the absolute HbA1c change from baseline after treatment, or achievement in a target HbA1c level [22]. Based on this report [22], we analyzed the glycemic effectiveness or responder in three ways; achievement in 1) absolute value of HbA1c reduction $\geq 0.6 \%$, 2) HbA1c $\leq 7.5 \%$, and 3) $\geq 9.8 \%$, of baseline HbA1c. Using effectiveness or responder definitions based on absolute reduction $\geq 0.6 \% \mathrm{HbA} 1 \mathrm{c}$, achievement in $\mathrm{HbA} 1 \mathrm{c} \leq 7.5 \%$, and $\geq 9.8 \%$, of baseline HbA1c reduction, the percentage rate in responders were $51.1 \%, 55.9 \%$ and $42.5 \%$, respectively. Regardless of definitions of responder, duration of diabetes, baseline FPG and HbA1c, and numbers of ADDs were significantly different between the responders and non-responders. However, the rest two variables are different according to the definitions of responder. Regarding clinical characteristics predicting in responsiveness of exenatide, shorter duration of diabetes was the only predictive variable for responders in glucose control. Those who had higher baseline HbA1c, a critical variable of potential confounder, showed the more reduction in absolute $\mathrm{HbAlc}$ and percentage of baseline $\mathrm{HbA1c}$, but the lower achievement in target HbA1c. Previous reports also showed the consistent results that the higher baseline $\mathrm{HbA1c}$ was related to the more HbA1c reduction but less probability of achievement in glycemic goal [22-24]. Regarding glycemic effectiveness in response to weight change, treatment with exenatide twice a day significantly reduced $-3.22 \mathrm{Kg}$ body weight. Of the participants who completed the study, 63.3\% $(n=369)$ experienced the both benefits of GLP-1 analogues on HbA1c reduction and weight loss. Those who got none benefits of exenatide were just $3.77 \%$. In the study, BMI did not affect glucose-lowering effect of exenatide. In one study which was aiming to investigate metabolic outcomes in patients with type 2 diabetes treated with exenatide, the response in $\mathrm{HbA1c}$ reduction was independent of baseline weight, and BMI [25]. Also, most subjects enrolled in this PMS study showed high BMI due to Korean insurance policy during the study period. This may have influenced the outcome of the study.

With respect to the adverse events of exenatide, of 181 participants who discontinued exenatide injection, 37\% ones failed to continue on exenatide within 8 weeks. Of 334 participants who discontinued exenatide injection between 8 weeks and 20 weeks, $14.7 \%$ of subjects discontinued on exenatide injection. Taken together, of 1269 subjects retrieved from safety analysis set, 161 (9.1\%) reported to stop the exenatide injection. Those who were analyzed for effectiveness analysis set $(n=754)$ at 20 weeks in this PMS OS, 22.9\% subjects reported any adverse events. Based on this finding, caution should be paid to the patients' tolerability.

The strengths of this study are the large sample number and the real-world clinical practice setting with patients of only Asian ethnic background. It, however, also has important weaknesses. This study did not provide the laboratory data to evaluate the pancreatic $\beta$-cell functions and peripheral insulin sensitivity which are the key pathophysiologic factors in the development and progress of T2DM. The clinical implications of duration of diabetes, baseline FPG and HbA1c, and numbers of ADDs which were different between the responders and non-responders might be explained in these assessments. Second, in the balanced stance of both studies design of RCTs and OS, this study might remain observer and selection bias which are reflected by not scientific milieu but socio-economic one. Underreporting also occurred especially in drug adverse events. A final major limitation is the drop out data from missing data and adherence to physicians' orders.

\section{Conclusions}

In conclusion, the results of the study of real-life clinical practice in Korea demonstrate that, in Korean subjects with T2DM, exenatide twice a day resulted significant reduction in glycemic control and relative tolerance in Korean patients with T2DM in real-world clinical setting.

\section{Additional file}

Additional file 1: Table S1. The institutions and ethic committee's reference number of this study. (DOCX $23 \mathrm{~kb}$ )

\begin{abstract}
Abbreviations
ADD: Anti-diabetic drug; BMI: Body mass index; Dipeptidyl peptidase-4: DPP4; FPG: Fasting plasma glucose; GLP-1: Glucagon-like-peptide-1; HbA1c: Hemoglobin A1c; OS: Observation study; PMS: Post-marketing surveillance; RCT: Randomized clinical trial; T2DM: Type 2 diabetes mellitus
\end{abstract}

\section{Acknowledgements}

Not applicable.

\section{Funding}

This work was supported by a grant from AstraZeneca. The funder managed the data, and its representative was involved in the data analysis and preparation of manuscript. The funder had no role in study design and decision to publish. 


\section{Availability of data and materials}

The datasets used and/or analyzed during the current study are available from the corresponding author on reasonable request.

\section{Authors' contributions}

J.-H.C. and B.-W.L. designed the study. A.K. performed the statistical analysis. Y.-C.H., J.-H.C., and B.-W.L. interpreted the data. A.K., E.J., and Y.Y. contributed to discussion. Y.-C.H., A.K., and B.-W.L. wrote the manuscript. Y.Y. edited and revised the manuscript. J.-H.C \& B.-W.L are the guarantors of this work and, as such, had full access to all the data in the study and takes responsibility for the integrity of the data and the accuracy of the data analysis. All authors read and approved the final manuscript.

\section{Ethics approval and consent to participate}

The study protocol was written in accordance with the requirement of Korean Ministry of Food and Drug Safety (MFDS) and reviewed by institutional review board (IRB)/independent ethics committee at each hospital before the initiation of study enrolment (Additional file 1: Table S1). All of patients provided written informed consents. The study was carried out according to the principles of the Declaration of Helsinki.

\section{Consent for publication}

Not applicable.

\section{Competing interests}

The authors declare that they have no competing interests.

\section{Publisher's Note}

Springer Nature remains neutral with regard to jurisdictional claims in published maps and institutional affiliations.

\section{Author details}

'Division of Endocrinology and Metabolism, Department of Internal Medicine, Kyung Hee University School of Medicine, Kyung Hee University Hospital at Gangdong, Seoul, South Korea. ${ }^{2}$ AstraZeneca, Seoul, South Korea. ${ }^{3}$ Division of Endocrinology and Metabolism, Department of Internal Medicine, Seoul St. Mary's Hospital, College of Medicine, The Catholic University of Korea, 222 Banpo-daero, Seoul, Seocho-gu 06591, South Korea. ${ }^{4}$ Division of Endocrinology and Metabolism, Department of Internal Medicine, Severance Hospital, Yonsei University College of Medicine, 50-1 Yonsei-Ro, Seoul, Seodaemun-Gu 03722, South Korea.

Received: 16 March 2017 Accepted: 16 October 2017

\section{Published online: 25 October 2017}

\section{References}

1. Chan JC, Malik V, Jia W, Kadowaki T, Yajnik CS, Yoon KH, et al. Diabetes in Asia: epidemiology, risk factors, and pathophysiology. JAMA. 2009;301:2129-40.

2. Kim DJ. The epidemiology of diabetes in Korea. Diabetes Metab J. 2011;35: 303-8.

3. Kim CS, Ko SH, Kwon HS, Kim NH, Kim JH, Lim S, et al. Prevalence, awareness, and management of obesity in Korea: data from the Korea national health and nutrition examination survey (1998-2011). Diabetes Metab J. 2014;38:35-43.

4. Jeon JY, Kim DJ, Ko SH, Kwon HS, Lim S, Choi SH, et al. Current status of glycemic control of patients with diabetes in Korea: the fifth Korea national health and nutrition examination survey. Diabetes Metab J. 2014;38:197-203.

5. Levetan C. Oral antidiabetic agents in type 2 diabetes. Curr Med Res Opin. 2007;23:945-52.

6. Rozenfeld Y, Hunt JS, Plauschinat C, Wong KS. Oral antidiabetic medication adherence and glycemic control in managed care. Am J Manag Care. 2008;14:71-5.

7. Gentilella R, Bianchi C, Rossi A, Rotella CM. Exenatide: a review from pharmacology to clinical practice. Diabetes Obes Metab. 2009;11:544-56.

8. Chon S, Gautier JF. An update on the effect of Incretin-based therapies on beta-cell function and mass. Diabetes Metab J. 2016;40:99-114.

9. Moretto TJ, Milton DR, Ridge TD, Macconell LA, Okerson T, Wolka AM, et al. Efficacy and tolerability of exenatide monotherapy over 24 weeks in antidiabetic drug-naive patients with type 2 diabetes: a randomized, double-blind, placebo-controlled, parallel-group study. Clin Ther. 2008:30:1448-60.
10. Buse JB, Henry RR, Han J, Kim DD, Fineman MS, Baron AD. Effects of exenatide (exendin-4) on glycemic control over 30 weeks in sulfonylureatreated patients with type 2 diabetes. Diabetes Care. 2004;27:2628-35.

11. DeFronzo RA, Ratner RE, Han J, Kim DD, Fineman MS, Baron AD. Effects of exenatide (exendin-4) on glycemic control and weight over 30 weeks in metformin-treated patients with type 2 diabetes. Diabetes Care. 2005;28: 1092-100.

12. Kendall DM, Riddle MC, Rosenstock J, Zhuang D, Kim DD, Fineman MS, et al. Effects of exenatide (exendin-4) on glycemic control over 30 weeks in patients with type 2 diabetes treated with metformin and a sulfonylurea. Diabetes Care. 2005;28:1083-91.

13. Zinman B, Hoogwerf BJ, Duran Garcia S, Milton DR, Giaconia JM, Kim DD, et al. The effect of adding exenatide to a thiazolidinedione in suboptimally controlled type 2 diabetes: a randomized trial. Ann Intern Med. 2007;146:477-85.

14. Kodama K, Tojjar D, Yamada S, Toda K, Patel CJ, Butte AJ. Ethnic differences in the relationship between insulin sensitivity and insulin response: a systematic review and meta-analysis. Diabetes Care. 2013;36:1789-96.

15. Kim YG, Hahn S, Oh TJ, Park KS, Cho YM. Differences in the HbA1c-lowering efficacy of glucagon-like peptide-1 analogues between Asians and nonAsians: a systematic review and meta-analysis. Diabetes Obes Metab. 2014;16:900-9.

16. Pratley RE. The efficacy and effectiveness of drugs for diabetes: how do clinical trials and the real world compare? Diabetologia. 2014;57:1273-5.

17. Guo XH. The value of short- and long-acting glucagon-like peptide-1 agonists in the management of type 2 diabetes mellitus: experience with exenatide. Curr Med Res Opin. 2016:32:61-76.

18. Ware $\mathrm{JH}$, Hamel MB. Pragmatic trials-guides to better patient care? N Engl J Med. 2011;364:1685-7.

19. Macconell L, Brown C, Gurney K, Han J. Safety and tolerability of exenatide twice daily in patients with type 2 diabetes: integrated analysis of 5594 patients from 19 placebo-controlled and comparator-controlled clinical trials. Diabetes Metab Syndr Obes. 2012;5:29-41.

20. Lee YH, Lee BW, Kwon HJ, Kang ES, Cha BS, Lee HC. Higher morning to evening ratio in total dose of twice-daily biphasic insulin analog might be effective in achieving glucose control in patients with poorly controlled type 2 diabetes. Diabetes Technol Ther. 2012;14:508-14.

21. Nikfar S, Abdollahi M, Salari P. The efficacy and tolerability of exenatide in comparison to placebo; a systematic review and meta-analysis of randomized clinical trials. J Pharm Pharm Sci. 2012:15:1-30.

22. Jones AG, Shields BM, Hyde CJ, Henley WE, Hattersley AT. Identifying good responders to glucose lowering therapy in type 2 diabetes: implications for stratified medicine. PLoS One. 2014;9:e111235.

23. Defronzo RA, Stonehouse AH, Han J, Wintle ME. Relationship of baseline $\mathrm{HbA1c}$ and efficacy of current glucose-lowering therapies: a meta-analysis of randomized clinical trials. Diabet Med. 2010;27:309-17.

24. Esposito K, Chiodini P, Capuano A, Maiorino MI, Bellastella G, Giugliano D. Baseline glycemic parameters predict the hemoglobin A1c response to DPP-4 inhibitors : meta-regression analysis of 78 randomized controlled trials with 20,053 patients. Endocrine. 2014;46:43-51.

25. Buysschaert M, Preumont V, Oriot PR, Paris I, Ponchon M, Scarniere D, et al. One-year metabolic outcomes in patients with type 2 diabetes treated with exenatide in routine practice. Diabetes Metab. 2010;36:381-8. 